\title{
Acute exacerbations and pulmonary hypertension in advanced idiopathic pulmonary fibrosis
}

\author{
Eoin P. Judge ${ }^{\star, \#}$, Aurelie Fabre
}

ABSTRACT: The aim of this study was to evaluate the risk factors for and outcomes of acute exacerbations in patients with advanced idiopathic pulmonary fibrosis (IPF), and to examine the relationship between disease severity and neovascularisation in explanted IPF lung tissue.

55 IPF patients assessed for lung transplantation were divided into acute $(n=27)$ and non-acute exacerbation $(n=28)$ groups. Haemodynamic data was collected at baseline, at the time of acute exacerbation and at lung transplantation. Histological analysis and CD31 immunostaining to quantify microvessel density (MVD) was performed on the explanted lung tissue of 13 transplanted patients.

Acute exacerbations were associated with increased mortality $(p=0.0015)$. Pulmonary hypertension $(\mathrm{PH})$ at baseline and acute exacerbations were associated with poor survival $(p<0.01)$. PH at baseline was associated with a significant risk of acute exacerbations (HR 2.217, $\mathbf{p}=\mathbf{0 . 0 4 1}$ ). Neovascularisation (MVD) was significantly increased in areas of cellular fibrosis and significantly decreased in areas of honeycombing. There was a significant inverse correlation between mean pulmonary artery pressure and MVD in areas of honeycombing.

Acute exacerbations were associated with significantly increased mortality in patients with advanced IPF. PH was associated with the subsequent development of an acute exacerbation and with poor survival. Neovascularisation was significantly decreased in areas of honeycombing, and was significantly inversely correlated with mean pulmonary arterial pressure in areas of honeycombing.

KEYWORDS: Acute exacerbation, idiopathic pulmonary fibrosis, pulmonary hypertension, pulmonary vascular, risk factors, survival

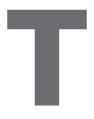

he natural history of idiopathic pulmonary fibrosis (IPF) has been described as a progressive decline in pulmonary function leading to death from respiratory failure or complicating comorbidity [1]. More recently, abrupt deteriorations of patients' clinical status leading to a more unpredictable clinical course have been acknowledged. These acute, clinically significant deteriorations of unknown cause in patients with underlying IPF have been defined as "acute exacerbations" [2]. Acute exacerbations are histologically characterised as diffuse alveolar damage (DAD) superimposed on underlying usual interstitial pneumonia (UIP) $[3,4]$.

The natural history of the impact of acute exacerbations on IPF patients remains to be further elucidated. Estimates of the incidence and mortality rate of acute exacerbations vary greatly, depending on the diagnostic criteria and follow-up period used $[3,5]$. Although there have been reports of acute respiratory deterioration occurring in patients with IPF after thoracic surgery [6] and bronchoalveolar lavage [3], there are currently no known risk factors for acute exacerbations. A recent study evaluated the incidence, risk factors and outcomes of acute exacerbations of patients with mildly severe IPF [7], but the impact of acute exacerbations on patients with advanced disease has not been evaluated. In addition, the potential association between pulmonary hypertension $(\mathrm{PH})$, a disease process which is increasingly recognised as having important implications in IPF patients [8], and subsequent development of an acute exacerbation has not been evaluated.

The relative roles played by neovascularisation and vascular regression in the pathogenesis of IPF remain uncertain. Previous histological studies have shown a heterogeneous pattern of vessel turnover, with the extent of both increased

\section{AFFILIATIONS}

${ }^{*}$ Depts of Respiratory Medicine, and "Pathology, Mater Misericordiae University Hospital, and

\#The Irish National Lung Transplant Unit, Mater Misericordiae University Hospital, Dublin, Ireland.

CORRESPONDENCE E.P. Judge

Dept of Respiratory Medicine, and the Irish National Lung Transplant Unit

Mater Misericordiae University Hospital

Eccles Street

Dublin 7

Ireland

E-mail: eoinjudge@yahoo.com

Received:

July 062011

Accepted after revision:

Nov 022011

First published online:

Dec 012011 
capillary density and vascular regression varying according to the extent of the tissue fibrosis that is present [9]. Consequently, the importance of considering disease severity when analysing these vascular changes has been acknowledged [10]. The relationship between neovascularisation and disease severity (in terms of histological severity of disease and pulmonary function) has been studied previously $[9,11]$. However, the relationship between these pulmonary microvascular changes and pulmonary arterial pressures $(\mathrm{Ppa})$ has not been evaluated.

Therefore, the purpose of this study was to evaluate the incidence, risk factors and outcomes of acute exacerbations in patients with advanced IPF awaiting lung transplantation, and to examine the relationship between $\mathrm{PH}$ at baseline and subsequent acute exacerbations. In addition, we examined the relationship between neovascularisation and disease severity (in terms of histological tissue type, pulmonary function and $\mathrm{PH})$ in the explanted lung tissue of patients with advanced IPF.

\section{METHODS}

\section{Subjects}

All IPF patients who were assessed for lung transplantation between 2005 and 2010 at the Irish National Lung Transplant Unit in the Mater Misericordiae University Hospital (Dublin, Ireland) were included in this study. 55 patients met the accepted diagnostic criteria for IPF. The study was approved by the Ethics and Audit Committee and informed consent was obtained from the patients. Acute exacerbations were defined as an acute onset of increased dyspnoea and hypoxia with progressive infiltrates on chest radiographs or high-resolution computed tomography (HRCT) of the thorax, within the preceding 30 days, in the absence of infection, pulmonary embolism or cardiac failure (as confirmed by clinical, radiological, echocardiographic and microbiological analysis) [2]. Patients who re-presented with a possible acute exacerbation during the study period were evaluated using a standardised diagnostic methodology which included a chest radiograph or HRCT of the thorax, white cell count, C-reactive protein, D-dimer level (with computed tomography pulmonary angiogram if indicated), sputum microbiological analysis and echocardiogram. Patients were sub-grouped according to the definition of acute exacerbation into those with $(n=27)$ and those without acute exacerbation $(n=28)$ (table 1). Patients were also sub-grouped into those who underwent lung transplantation over the follow-up period $(n=13)$ and those who did not $(n=42)$.

The Irish National Lung Transplant database and patient charts provided the data set. Baseline patient demographics (age, sex, blood group, cytotoxic antibody status and body mass index) were recorded. Pulmonary function testing (including forced expiratory volume in $1 \mathrm{~s}$ (FEV1), forced vital capacity (FVC), total lung capacity and diffusing capacity of the lung for carbon monoxide $(\mathrm{DL}, \mathrm{CO})$ ) was performed on all patients who were assessed for lung transplantation. Transthoracic echocardiographic data (left ventricular ejection fraction and right ventricular systolic pressure) were collected at the time of initial lung transplant assessment and during an acute exacerbation. Right heart catheterisation (RHC) data $(P$ pa, pulmonary capillary wedge pressure $(P \mathrm{pcw})$ and cardiac output) was collected at the time of initial transplant assessment and also at the time of lung transplantation. $\mathrm{PH}$ was defined as a mean $P$ pa $(\mathrm{mPpa}) \geqslant 25 \mathrm{mmHg}$ on RHC. The explanted lung tissue from the 13 lung transplant recipients underwent blinded histological assessment (A. Fabre) for evidence of pulmonary vascular changes and DAD.

\section{Histological analysis}

Microvessel density (MVD; or neovascularisation) was quantified for cases using CD31 immunostaining. Paraffin embedded

TABLE 1 Baseline characteristics at initial assessment

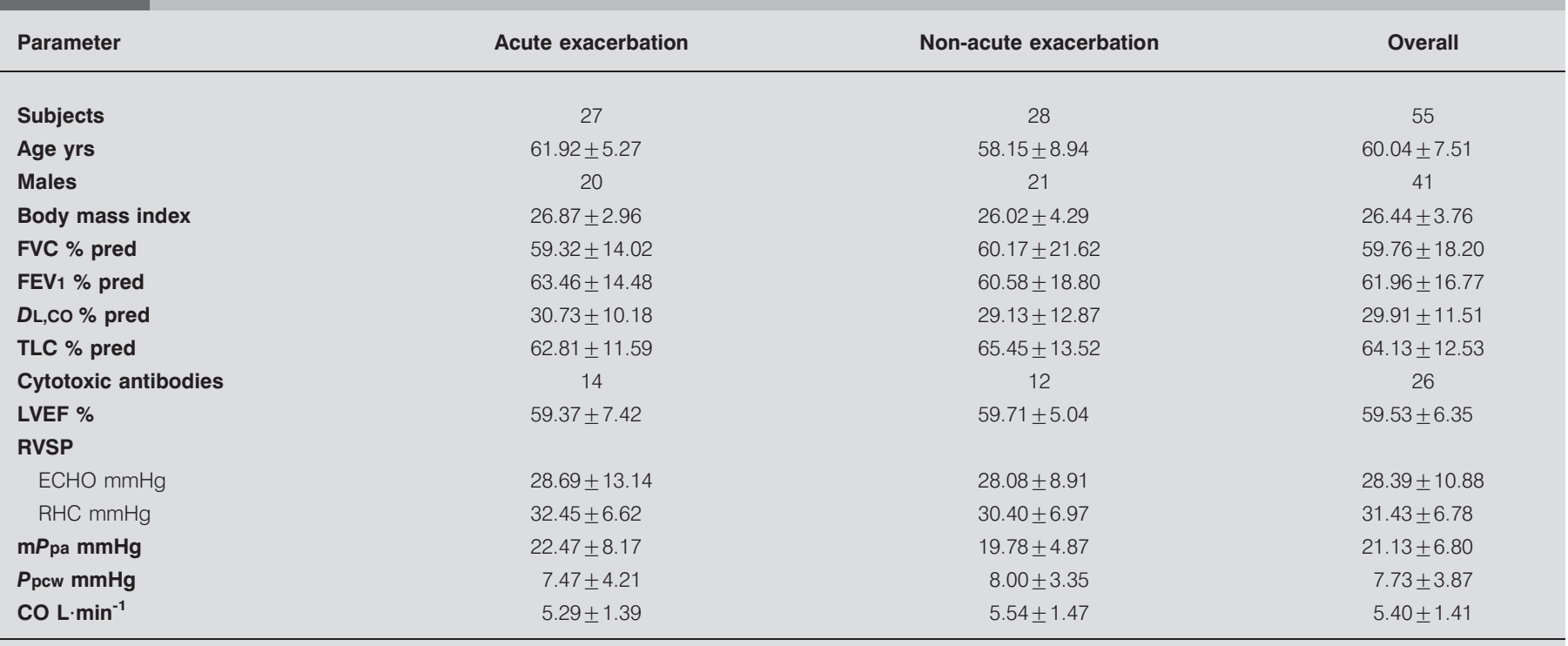

Data are presented as $\mathrm{n}$ or mean \pm SD. FVC: forced vital capacity; \% pred: \% predicted; FEV1: forced expiratory volume in $1 \mathrm{~s}$; $D L, C 0$ : diffusing capacity of the lung for carbon monoxide; TLC: total lung capacity; LVEF: left ventricular ejection fraction; RVSP: right ventricular systolic pressure; RHC: right heart catheterisation; $\mathrm{mPpa:} \mathrm{mean}$ pulmonary arterial pressure; Ppcw: pulmonary capillary wedge pressure; CO: cardiac output. 
4- $\mu \mathrm{m}$ sections were deparaffinised and processed on an automatic Ventana Benchmark XT Immunostainer (Ventana Medical Systems Inc., Tucson AZ, USA). They were then subjected to antigen retrieval (Ventana Protease for $12 \mathrm{~min}$ ) and incubated with antibody CD31 (ref M0823, dilution 1:40; Dako Diagnostics Ireland Ltd, Dublin, Ireland) for $32 \mathrm{~min}$ at $37^{\circ} \mathrm{C}$. Antibody detection was performed using an UltraView Universal DAB Detection Kit (Ventana Medical Systems Inc).

As UIP presents a heterogeneous distribution of fibrosis, eight different fields at a magnification of $\times 20$ were assessed in the explanted lung of each transplant recipient, including significant/cellular interstitial fibrosis (four fields) as previously described, and honeycomb areas (four fields) [12]. Architecturally preserved lung tissue present in the same explanted lung was used as an internal "normal" control.

Digital images of CD31 stained lung fibrosis were captured with a Nikon Digital Sight DS-5M camera (Nikon Instruments Europe BV, Surrey, UK), and total tissue area stained with CD31 was quantified using Image $J$ software (National Institutes of Health, Bethesda, MD, USA). MVD was assessed on inverted and binary images, and the percentage of area covered by vessels in areas of fibrosis, honeycomb and control was calculated for each case, as previously described [13]. Mean values for MVD were then calculated by averaging the percentage area covered by vessels.

\section{Statistical analysis}

Baseline characteristics were summarised for comparison purposes by calculating the mean and SD. Baseline demographic data, pulmonary function, echocardiographic data and RHC data of the acute and non-acute exacerbation groups were compared using the Mann-Whitney test. Interval changes in echocardiogram and RHC data were assessed using the Wilcoxon test. A Cox regression analysis was used to identify significant variables capable of predicting an acute exacerbation or acting as prognostic factors. Survival between the acute and non-acute exacerbation groups was evaluated using a Kaplan-Meier survival curve and the log-rank test. Spearman rank correlation was used as a non-parametric measure of correlation between echocardiographic and RHC data, and between histological and $P$ pa data.

All tests were two-tailed and a p-value $<0.05$ was taken to represent statistical significance. All statistical analysis was performed using SPSS software (SPSS 16.0.2; SPSS Inc., Chicago, IL, USA).

\section{RESULTS}

\section{Baseline characteristics}

The baseline characteristics of the 55 IPF patients who were included in this study are presented in table 1. Baseline characteristics of patients with $(n=27)$ and without $(n=28)$ acute exacerbation are also shown. There was no statistically significant difference in demographic, pulmonary function or cardiovascular data between both groups.

\section{Incidence and effects of acute exacerbation on patient survival}

The incidence of acute exacerbation was $19.11 \%$ per annum. As lung transplantation alters the natural course of IPF,

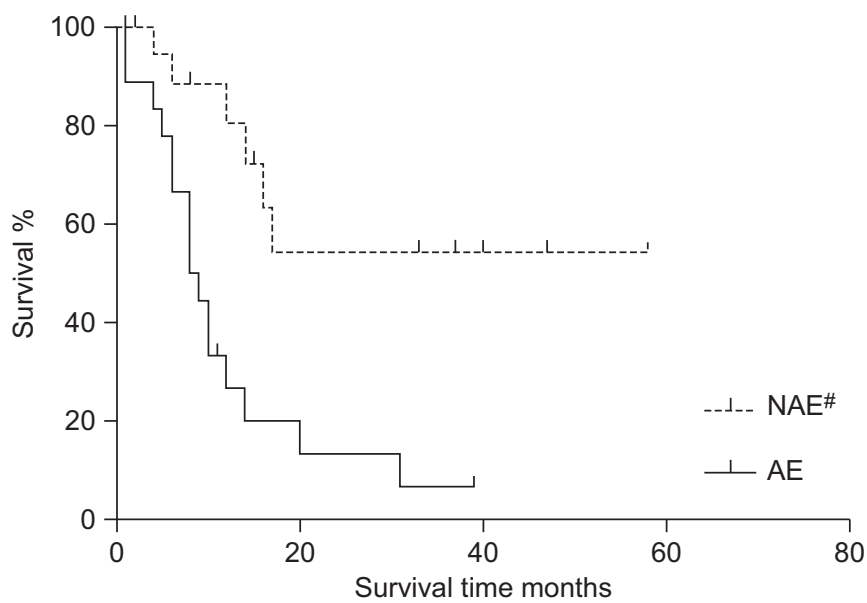

FIGURE 1. Survival of patients in the acute and non-acute exacerbation groups (AE and NAE, respectively). As lung transplantation alters the natural course of idiopathic pulmonary fibrosis, 13 transplantation events over the follow-up period were censored when performing this Kaplan-Meier survival analysis. ${ }^{*}: p=0.0015$.

13 transplantation events over the follow-up period were censored when performing survival analysis. Acute exacerbations were associated with a significant increase in mortality in these patients, with 16 patients dying in the acute exacerbation group and six patients dying in the non-acute exacerbation group ( $\mathrm{p}=0.0015$ ) (fig. 1). The majority of patients died during an acute exacerbation $(\mathrm{n}=11,68.75 \%)$. The median time

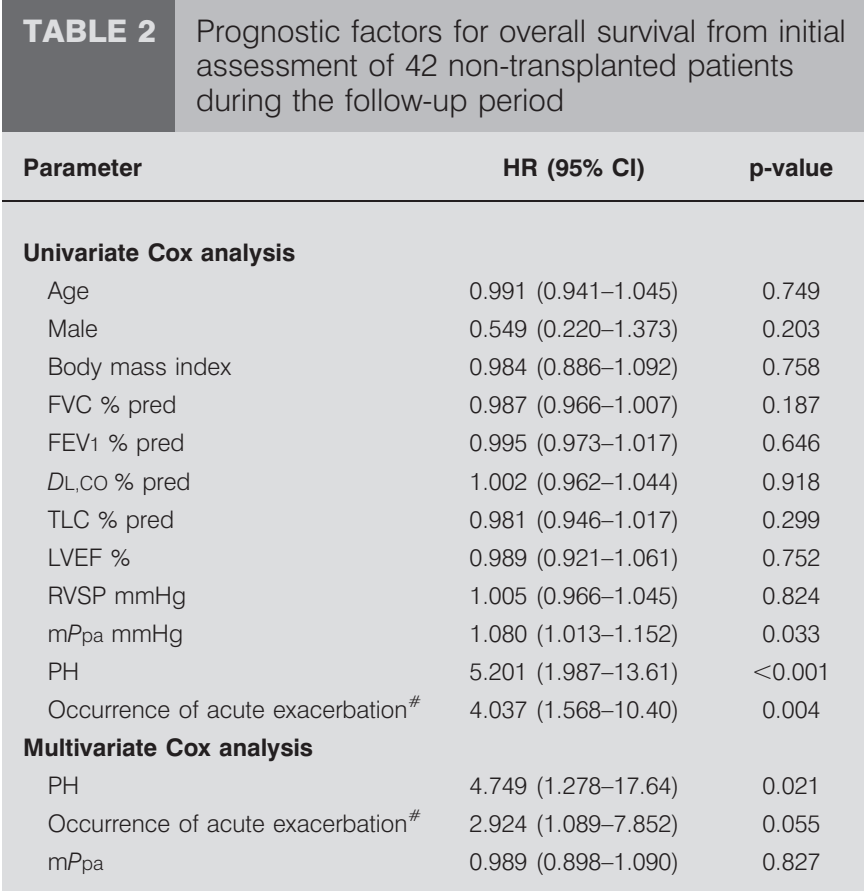

FVC: forced vital capacity; \% pred: \% predicted; FEV1: forced expiratory volume in $1 \mathrm{~s}$; $D$ L,CO: diffusing capacity of the lung for carbon monoxide; TLC: total lung capacity; LVEF: left ventricular ejection fraction; RVSP: right ventricular systolic pressure; $\mathrm{mPpa}$ : mean pulmonary arterial pressure; $\mathrm{PH}$ : pulmonary hypertension. * : overall occurrence during follow-up period. 


\begin{tabular}{|c|c|c|c|}
\hline \multirow{2}{*}{$\begin{array}{l}\text { TABLE } 3 \\
\text { Parameter }\end{array}$} & \multicolumn{3}{|c|}{$\begin{array}{l}\text { Risk factors at initial assessment for acute } \\
\text { exacerbation }\end{array}$} \\
\hline & & HR (95\% Cl) & $\mathrm{p}$-value \\
\hline \multicolumn{4}{|c|}{ Univariate Cox analysis } \\
\hline Age & & $1.023(0.967-1.081)$ & 0.435 \\
\hline Male & & $0.599(0.250-1.434)$ & 0.253 \\
\hline Body mass & ndex & $1.043(0.939-1.159)$ & 0.437 \\
\hline FVC \% pred & & 0.999 (0.979-1.019) & 0.928 \\
\hline FEV $1 \%$ prec & & $1.008(0.987-1.031)$ & 0.438 \\
\hline$D\llcorner, C O \%$ pre & & $1.003(0.967-1.041)$ & 0.873 \\
\hline TLC \% pred & & $0.982(0.946-1.019)$ & 0.347 \\
\hline LVEF \% & & $0.989(0.922-1.062)$ & 0.767 \\
\hline RVSP $\mathrm{mmH}$ & & $1.010(0.977-1.042)$ & 0.597 \\
\hline $\mathrm{mPpa} \mathrm{mmHg}$ & & $1.043(0.977-1.114)$ & 0.210 \\
\hline $\mathrm{PH}$ & & $2.217(1.005-4.889)$ & 0.041 \\
\hline Ppcw mmHg & & $0.938(0.843-1.044)$ & 0.241 \\
\hline \multicolumn{4}{|c|}{ Multivariate Cox analysis } \\
\hline Male & & $0.587(0.398-1.139)$ & 0.182 \\
\hline $\mathrm{PH}$ & & 2.510 (1.119-5.628) & 0.026 \\
\hline
\end{tabular}

FVC: forced vital capacity; \% pred: \% predicted; FEV1: forced expiratory volume in $1 \mathrm{~s} ; D L, C O$ : diffusing capacity of the lung for carbon monoxide; TLC: total lung capacity; LVEF: left ventricular ejection fraction; RVSP: right ventricular systolic pressure; $\mathrm{mPpa}$ : mean pulmonary arterial pressure; $\mathrm{PH}$ : pulmonary hypertension; $P$ pcw: pulmonary capillary wedge pressure.

interval from initial assessment to acute exacerbation in patients who did not undergo lung transplantation was 6 months, and the median survival of these patients postacute exacerbation was 1 month.

Table 2 shows the prognostic impact of factors on the survival of patients who did not undergo lung transplantation over the study period. Cox univariate analysis revealed significant associations between occurrence of acute exacerbations over the study period (HR 4.0374, 95\% CI 1.568-10.399; $\mathrm{p}=0.004$ ), baseline $\mathrm{mPpa}$ (HR 1.080, 95\% CI 1.013-1.152; $\mathrm{p}=0.033)$ and $\mathrm{PH}$ at baseline (HR 5.201, 95\% CI 1.987-13.609; $\mathrm{p}=0.0008$ ), and poor overall survival. Multivariate Cox analysis showed that occurrence of acute exacerbation (HR 2.924, 95\% CI

\begin{tabular}{lcc} 
TABLE 4 & $\begin{array}{l}\text { Patient characteristics } \\
\text { transplantation }\end{array}$ \\
Subjects & 13 \\
Age & $57.69 \pm 5.42$ \\
Males & 10 \\
Body mass index & $26.73 \pm 3.02$ \\
Cytotoxic antibodies & 6 \\
LVEF \% & $60.73 \pm 5.16$ \\
mPpa $\mathbf{~ m H g}$ & $36.21 \pm 16.73$ \\
Ppcw $\mathbf{~ m m H g}$ & $8.54 \pm 4.68$ \\
\hline
\end{tabular}

Data are presented as $\mathrm{n}$ or mean $\pm \mathrm{SD}$. LVEF: left ventricular ejection fraction; $\mathrm{mPpa}$ : mean pulmonary arterial pressure; Ppcw: pulmonary capillary wedge pressure.

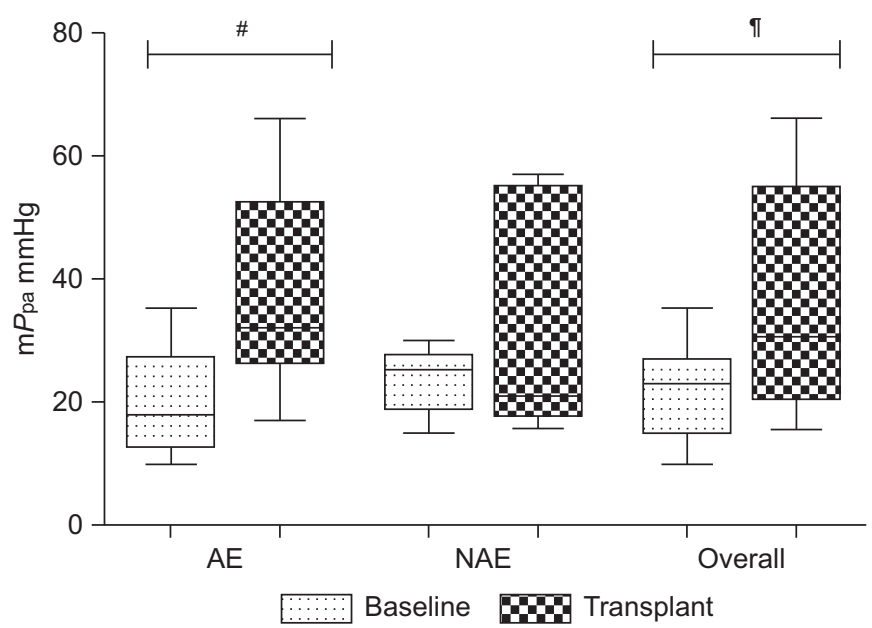

FIGURE 2. Mean pulmonary arterial pressure $(\mathrm{mPpa})$ in lung transplant recipients (acute exacerbation (AE): $n=8$; non-acute exacerbation (NAE): $n=5$; overall: $n=13$ ) at baseline and at the time of transplantation. ${ }^{*}: p=0.039, m P p a$ at baseline compared to $\mathrm{mPpa}$ at time of transplantation in transplant recipients who experienced an $\mathrm{AE} ;{ }^{\bullet}: \mathrm{p}=0.027, \mathrm{mPpa}$ at baseline compared to $\mathrm{mPpa}$ at time of transplantation in all transplant recipients. The median (interquartile range) of $\mathrm{mPpa}$ at baseline and at time of transplant, respectively, were as follows. AE: 18.00 (12.75-27.50) and 32.17 (26.42-52.42) mmHg; NAE: 25.33 (19.00-27.70) and 21.00 (17.84-55.17) mmHg; overall: $23.00(15.00-27.00)$ and $30.67(20.50-$ 55.00) $\mathrm{mmHg}$.

1.089-7.852; $\mathrm{p}=0.0342)$ and PH (HR 4.749, 95\% CI 1.278-17.641; $\mathrm{p}=0.0206)$ were associated with poorer overall survival during the study period.

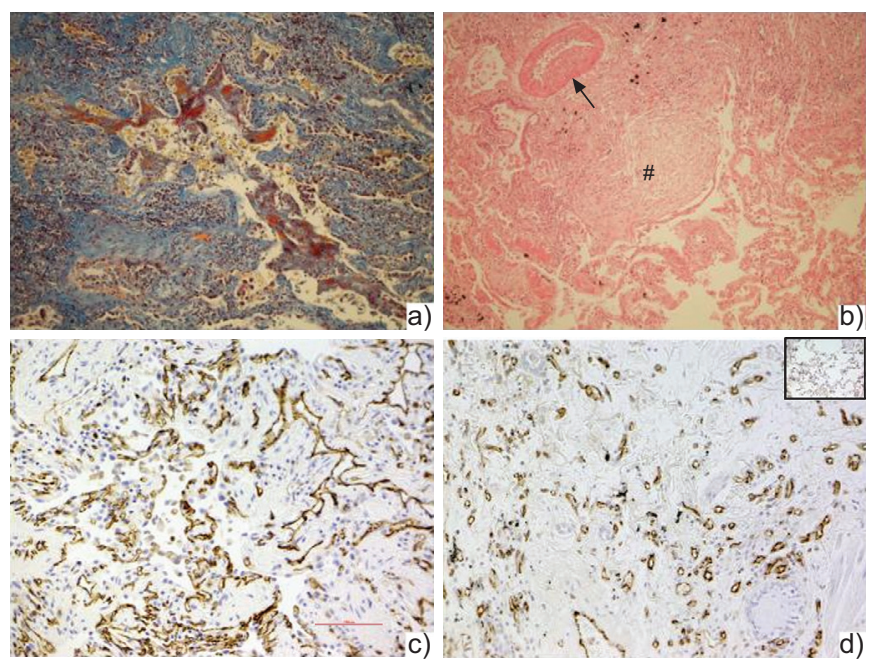

FIGURE 3. Explanted lung histology of: a) an idiopathic pulmonary fibrosis (IPF) lung showing diffuse alveolar damage with hyaline membranes lining the alveolar spaces, as seen in acute exacerbations (Martius Scarlet Blue stain); b) a branch of the pulmonary artery (arrow) adjacent to fibroblastic foci (\#) showing asymmetrical intimal thickening as seen in pulmonary hypertension (Haematoxylin and eosin stain); c and d) CD31 staining showing increased number of capillaries in the IPF lung and honeycomb areas compared to normal control (insert). Scale $\mathrm{bar}=100 \mu \mathrm{m}$ 


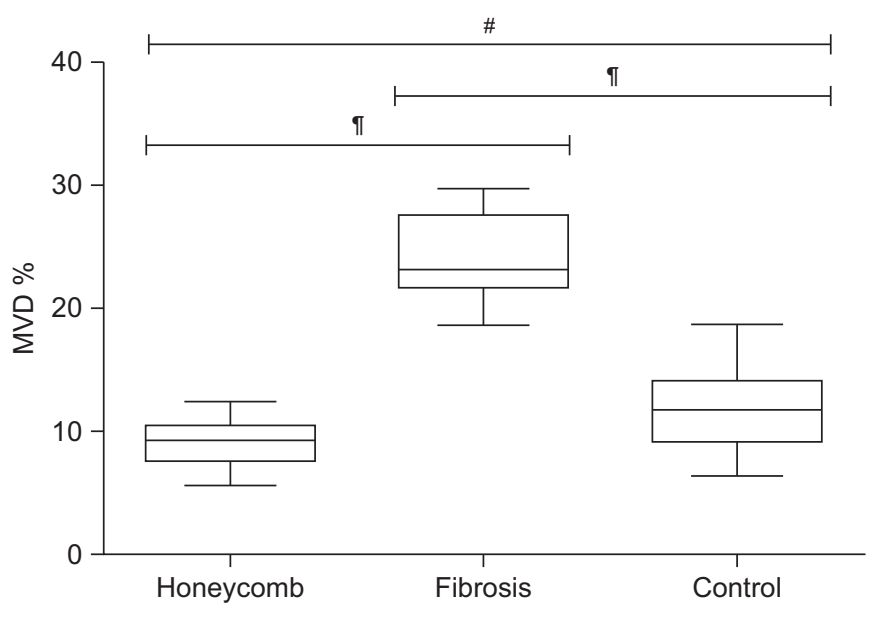

FIGURE 4. Mean microvessel density (MVD) in control (non-diseased) areas in areas of cellular fibrosis and in areas of honeycombing. ${ }^{*}: p=0.04$, MVD in areas of honeycomb compared to control areas; " $"$ : $p<0.0001$, MVD in areas of cellular fibrosis compared to control areas and MVD in areas of cellular fibrosis compared to areas of honeycomb. The median (interquartile range) of MVD was as follows. Honeycomb: 9.29 (7.54-10.47)\%; cellular fibrosis: 23.14 (21.68-27.57)\%; control: $11.74(9.14-14.11) \%$

\section{Pulmonary hypertension}

$\mathrm{PH}$ at baseline was associated with a significant risk of acute exacerbation (HR 2.217, 95\% CI 1.005-4.889; $p=0.041$ ), with 12 out of the 17 patients who had $\mathrm{PH}$ at baseline subsequently experiencing an acute exacerbation during follow-up period. There were no other significant associations between baseline variables and development of an acute exacerbation (table 3).

Mean right ventricular systolic pressure (RVSP) on echocardiogram increased significantly from $28.69 \pm 13.14 \mathrm{mmHg}$ at baseline to $37.00 \pm 15.64 \mathrm{mmHg}$ at the time of acute exacerbation $(n=27, p=0.0025)$. Mean left ventricular ejection fraction (LVEF) remained unchanged, with a mean LVEF of $59.37 \pm 7.42 \%$ at assessment and $60.03 \pm 8.85 \%$ at the time of acute exacerbation $(n=27, p=0.529)$. There was significant

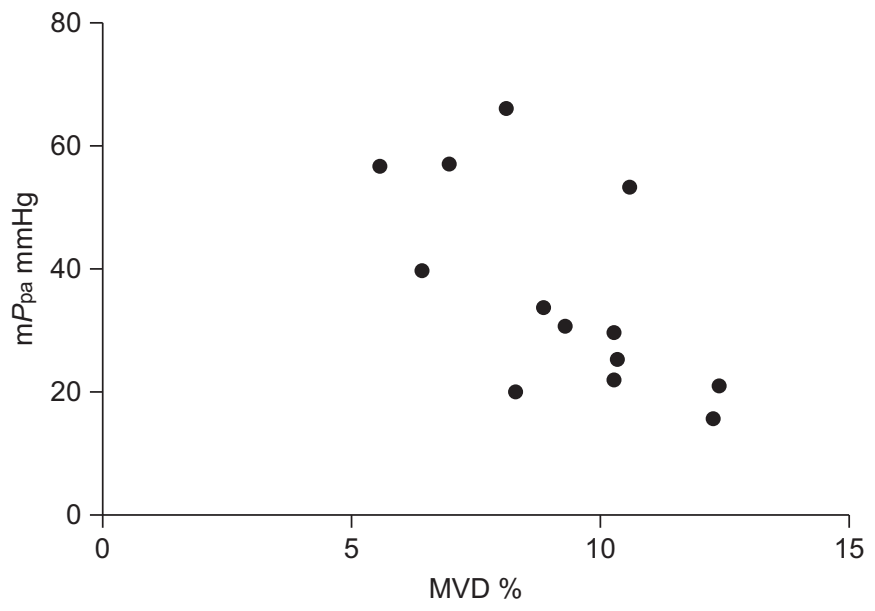

FIGURE 5. The relationship between mean microvessel density (MVD) in areas of honeycombing and mean pulmonary arterial pressure ( $\mathrm{mPpa}$ ). Spearman correlation rho $=-0.637 . p=0.019$. correlation between overall baseline RVSP measured by echocardiogram and RHC (rho $=0.41, p=0.0069)$. Correlation between baseline RVSP measured by echocardiogram and RHC for the AE group was also significant (rho=0.44, $\mathrm{p}=0.039)$, and approached significance for the non-acute exacerbation group $(\mathrm{rho}=0.38, \mathrm{p}=0.096)$.

\section{Lung transplantation}

$13(23.64 \%)$ of the 55 patients in this study underwent lung transplantation over the follow-up period. All lung transplants were single organ transplants. None of the 13 patients who received lung transplants died during the follow-up period. The characteristics of patients at the time of lung transplant are shown in table 4 .

$\mathrm{m} P$ pa increased significantly from $21.39 \mathrm{mmHg}$ at baseline to $36.21 \mathrm{mmHg}$ at the time of transplant $(\mathrm{n}=13, \mathrm{p}=0.027)$ (fig. 2$)$. $\mathrm{mPpa}$ also increased significantly from $20.92 \mathrm{mmHg}$ at initial assessment to $37.96 \mathrm{mmHg}$ at the time of transplant in the acute exacerbation group $(n=8, p=0.039)$. The increase in $\mathrm{mPpa}$ from $22.33 \mathrm{mmHg}$ to $33.40 \mathrm{mmHg}$ in the non-acute exacerbation group was not statistically significant $(n=5, p=0.887)$ (fig. 2). P pcw was similar at baseline $(7.64 \mathrm{mmHg})$ and at the time of transplant $(8.54 \mathrm{mmHg})$.

\section{Histological analysis of explanted lung tissue}

There was histological evidence of pulmonary vascular changes in the explanted lung tissue of all 13 patients who underwent lung transplantation (fig. 3b). The histological pattern of UIP was found in 11 cases, and DAD was found in four cases (fig. 3a). In three of these four cases the patients were being treated for an acute exacerbation at the time of lung transplantation.

MVD was significantly increased in cellular fibrotic areas compared to both control $(p<0.001)$ and honeycomb areas $(\mathrm{p}<0.001)$ (fig. 4), while MVD was significantly decreased in honeycomb areas compared to control areas $(p=0.04)$. There was a significant inverse correlation between $\mathrm{m} P$ pa and MVD in honeycomb areas (rho $=-0.637, p=0.019)$ (fig. 5) but not in cellular fibrotic areas (rho $=-0.258, \mathrm{p}=0.394$ ) (fig. 6). $\mathrm{PH}$ at the

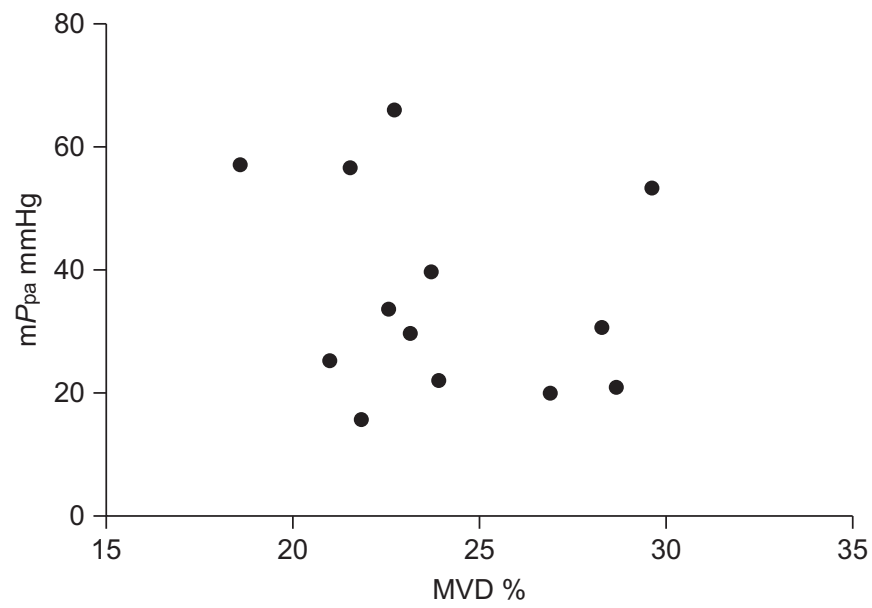

FIGURE 6. The relationship between mean microvessel density (MVD) in areas of cellular fibrosis and mean pulmonary arterial pressure (mPpa). Spearman correlation rho $=-0.258 . p=0.394$. 
time of lung transplantation did not correlate significantly with MVD in cellular fibrotic areas (rho $=-0.312, \mathrm{p}=0.299)$ or MVD in honeycomb areas (rho $=-0.445, \mathrm{p}=0.127$ ). Pulmonary function (FVC and DL,CO) did not correlate significantly with MVD in cellular fibrotic or honeycomb areas. There was no significant difference in MVD in cellular fibrotic or honeycomb areas in explanted lung tissue with histological evidence of DAD compared to tissue without evidence of DAD.

\section{DISCUSSION}

In this study, we found that acute exacerbations are common and are associated with significantly increased mortality in patients with advanced IPF. PH at baseline was associated with the subsequent development of an acute exacerbation and with poor overall survival, and $\mathrm{mP}$ pa was shown to increase significantly over time in patients who had experienced an acute exacerbation. We have also shown that neovascularisation is significantly increased in areas of cellular fibrosis and significantly decreased in honeycomb areas, and that there is a significant inverse relationship between $\mathrm{mP}$ pa and neovascularisation in areas of honeycombing.

Reports on the incidence and mortality from acute exacerbations of IPF have varied greatly [3,5]. This is probably due to differences in study design, the definition of acute exacerbation used and the severity of the disease. In a recent retrospective review of 461 patients with IPF (limited disease with a mean baseline $\mathrm{FVC} \geqslant 72 \%$ predicted and mean baseline $D \mathrm{~L}, \mathrm{CO}$ $\geqslant 62 \%$ pred), the $1-y r$ incidence of acute exacerbation was $11.6 \%$, and the median survival post-onset of acute exacerbation was 2.2 months [7]. In contrast to that study, patients in our study had advanced IPF, and acute exacerbation was associated with a slightly higher incidence and a lower median survival post-acute exacerbation. Therefore, our study suggests that acute exacerbations are more common and have an even poorer outcome in patients with advanced IPF. Previous reports have also indicated that a steady decline in pulmonary function is the major manifestation of disease progression in IPF $[14,15]$. The short time interval from initial assessment to acute exacerbation seen in this study, coupled with the poor outcome following acute exacerbation, suggests that acute exacerbation may also contribute to, and be a manifestation of, disease progression. It also emphasises the importance of urgent lung transplantation for these patients.

In this study, both univariate and multivariate logistic analysis indicates that $\mathrm{PH}$ is an independent predictor for development of an acute exacerbation in patients with advanced IPF. Risk factors for acute exacerbation are largely unknown. One recent study which looked at risk factors for developing an acute exacerbation, did not evaluate $\mathrm{PH}$ as a potential predictor [7]. In that study, nonsmokers with lower lung function were found to be at an increased risk of acute exacerbation. However, patients in that study had only mildly severe disease. This contrasted to our study where all patients had severe IPF and had been referred for lung transplantation [7]. Indeed, previous studies of patients with IPF referred for lung transplantation have shown that lung function is not predictive of survival in this cohort of patients with advanced disease [16, 17].

$\mathrm{PH}$ has been shown to be common in patients with IPF. Previous studies have shown a prevalence of $32-46 \%$ in patients with advanced disease $[8,18-20]$. The incidence of $\mathrm{PH}$ and the $\mathrm{mP}$ pa have also been shown to increase over time in patients undergoing lung transplantation [18], and the presence of $\mathrm{PH}$ has been associated with an increased risk of mortality in IPF patients with advanced disease [8, 21, 22]. In this study, $\mathrm{PH}$ at baseline (in addition to occurrence of acute exacerbation over the follow-up period) was found to be an independent predictor of increased mortality. Furthermore, both the number of patients with $\mathrm{PH}$ and the $\mathrm{mP}$ pa increased over time in both the acute and non-acute exacerbation groups at the time of transplant, and the increase in $\mathrm{mPpa}$ was statistically significant in the acute but not in the non-acute exacerbation group. Given the progression of $P$ pa over time, and the high incidence of $\mathrm{PH}$ and its association with acute exacerbation, these results support the hypothesis that patients with advanced IPF should be evaluated for occult PH. This data also provides further evidence for considering pulmonary vasodilator therapy in these patients $[23,24]$.

The effect of acute exacerbations of IPF on $P_{\mathrm{pa}}$ is largely unknown. Previous studies on a small number of patients suggest that mean RVSP is elevated during acute exacerbation [4], and that $\mathrm{PH}$ (as evidenced by histological findings) is implicated in disease progression or acute exacerbation [25]. In our study, mean RVSP increased significantly from baseline to the time of acute exacerbation while mean LVEF remained stable. $\mathrm{mP}$ pa also increased significantly over time in the acute exacerbation group. It is not clear whether $\mathrm{PH}$ is a causative factor itself or whether it results from acute exacerbation and disease progression. Indeed, IPF with PH may represent a distinct clinical phenotype [1]. The role of pulmonary vasodilator therapies in the treatment of acute exacerbations also remains to be established.

It is uncertain as to whether the primary vascular abnormality in IPF results from an increase or a reduction in neovascularisation. Establishing if neovascularisation plays a key role in abnormal extracellular matrix remodelling may have important therapeutic implications. Previous studies have shown that both increased capillary density and vascular regression occur in IPF, and indeed vary according to the extent of the disease $[9,26]$. In contrast to previous studies where tissue from non-fibrotic/disease-free lungs was used as controls $[9,12]$, our study used "normal" (i.e. non-diseased) lung tissue from within the same lung as controls. The authors believe that this is an important consideration in order to study the welldescribed heterogeneous pattern of both fibrosis and vascularisation throughout the whole lung.

The results of our study support the results of previous studies which show a heterogeneous pattern of neovascularisation according to disease severity $[9,26]$. Neovascularisation was significantly increased in areas of cellular fibrosis (areas of increased extracellular matrix where neovascularisation is composed of numerous capillaries and small, thin-walled vessels), and significantly decreased in honeycomb areas (less cellular areas with dense collagenous tissue). The increased MVD in less fibrotic areas may represent a compensatory response to hypovascularity in areas of dense fibrosis (honeycomb areas) [10]. Alternatively, it may be actively involved in the fibrogenic process [10], or may be involved in tissue regeneration [9]. Further studies are required to answer this question. 
Another interesting finding of this study was that $\mathrm{m} P$ pa at the time of lung transplantation was significantly inversely correlated with MVD in honeycomb areas but not with areas of cellular fibrosis. In a previous study which evaluated the relationship between $\mathrm{mP}$ pa and pulmonary venous changes in explanted IPF tissue, no significant correlation was found [27]. The findings in our study (which evaluates both arterial and venous changes) may be explained by the differences in vascular changes observed in differing fibrotic areas. In honeycomb areas, vascular changes were the same as those observed in idiopathic pulmonary arterial hypertension, with medial hypertrophy and intimal fibrosis of the muscular arteries and intimal fibrosis of the pulmonary veins [28]. These vessels may contribute most significantly to increased pulmonary vascular resistance, while the neovascularisation seen in cellular fibrotic areas may represent an adaptive proliferative change in response to altered vascular resistance rather than a primary event. The pathogenesis of $\mathrm{PH}$ in the setting of pulmonary fibrosis is believed to relate to the effect of alveolar hypoxia with subsequent vascular remodelling, and to the reduction of the vascular bed by fibrosis [29]. Our results support the role of this latter factor in the pathogenesis of $\mathrm{PH}$ in IPF. Alternatively, our results may suggest that honeycomb remodelling may play a major role in vascular resistance in IPF. Further studies are therefore required to evaluate these hypotheses. Pulmonary function has been shown to be a poor surrogate of pulmonary vascular disease in IPF, and this may explain the lack of correlation between pulmonary function measures and MVD seen in this study [19].

In addition to its retrospective design, the main limitation of this study relates to the cohort of IPF patients used, namely patients with advanced disease. Histological analysis was also performed on explanted IPF tissue pre-lung transplantation. Therefore, these results cannot be extended to all patients with IPF. Echocardiography was also used to assess haemodynamic changes during acute exacerbations. Although RHC is the only definitive method of diagnosing $\mathrm{PH}$, higher right ventricular systolic pressures on echocardiogram are suggestive of pulmonary hypertension, and RHC is recommended in these cases [30]. Given the obvious difficulties in performing RHCs (and other potential surrogate markers such as 6-min walk tests and $\mathrm{DL}, \mathrm{CO})$ in acutely unwell patients, echocardiography is currently the most practical method for assessing $P$ pa during acute exacerbations, and significant increases in RVSP as seen in this study are suggestive of $P$ pa [31].

Another potential limitation relates to the differences in the methodology of RHC at initial assessment and at the time of transplantation. Baseline RHC was performed while in an awake state in the cardiac catheterisation laboratory. Transplant RHC was performed under general anaesthesia in the operating room. The induction agents used in general anaesthesia may reduce $P$ pa [32]. Therefore, the $m P$ pa readings obtained under general anaesthesia at the time of transplant may have been underestimated. Furthermore, results from a previous study using similar RHC methodology support this method of comparing Ppa under these clinical circumstances [18]. Although we did not have a full set of haemodynamic variables at the time of transplantation and were therefore unable to assess for a serial change in cardiac output, the close similarity between baseline and transplant $P_{\mathrm{pcw}}$ suggests that the anaesthetic induction agents also had little effect on left ventricular function (which could have confounded $P$ pa results).

In conclusion, this study has shown that acute exacerbations are common and are associated with significantly increased mortality in patients with advanced IPF. PH at baseline was associated with the subsequent development of an acute exacerbation and with poor overall survival, and $\mathrm{mPpa}$ was shown to increase significantly over time in patients who had experienced an acute exacerbation. We have also shown that neovascularisation was significantly increased in areas of cellular fibrosis and significantly decreased in areas of honeycombing, and that $\mathrm{mPpa}$ was significantly inversely correlated with neovascularisation in areas of honeycombing.

\section{STATEMENT OF INTEREST}

None declared.

\section{REFERENCES}

1 Raghu G, Collard HR, Egan JJ, et al. An official ATS/ERS/JRS/ ALAT statement: idiopathic pulmonary fibrosis: evidence-based guidelines for diagnosis and management. Am J Respir Crit Care Med 2011; 183: 788-824.

2 Collard HR, Moore BB, Flaherty KR, et al. Acute exacerbations of idiopathic pulmonary fibrosis. Am J Respir Crit Care Med 2007; 176: 636-643.

3 Kim DS, Park JH, Park BK, et al. Acute exacerbation of idiopathic pulmonary fibrosis: frequency and clinical features. Eur Respir $J$ 2006; 27: 143-150.

4 Parambil JG, Myers JL, Ryu JH. Histopathologic features and outcome of patients with acute exacerbation of idiopathic pulmonary fibrosis undergoing surgical lung biopsy. Chest 2005; 128: 3310-3315.

5 Homma S, Sakamoto S, Kawabata M, et al. Cyclosporin treatment in steroid-resistant and acutely exacerbated interstitial pneumonia. Intern Med 2005; 44: 1144-1150.

6 Kondoh Y, Taniguchi H, Kitaichi M, et al. Acute exacerbation of interstitial pneumonia following surgical lung biopsy. Respir Med 2006; 100: 1753-1759.

7 Song JW, Hong SB, Lim CM, et al. Acute exacerbation of idiopathic pulmonary fibrosis: incidence, risk factors and outcome. Eur Respir J 2011; 37: 356-363.

8 Lettieri CJ, Nathan SD, Barnett SD, et al. Prevalence and outcomes of pulmonary arterial hypertension in advanced idiopathic pulmonary fibrosis. Chest 2006; 129: 746-752.

9 Ebina M, Shimizukawa M, Shibata N, et al. Heterogeneous increase in CD34-positive alveolar capillaries in idiopathic pulmonary fibrosis. Am J Respir Crit Care Med 2004; 169: 1203-1208.

10 Renzoni EA. Neovascularization in idiopathic pulmonary fibrosis: too much or too little? Am J Respir Crit Care Med 2004; 169: 1179-1180.

11 Parra ER, David YR, da Costa LR, et al. Heterogeneous remodeling of lung vessels in idiopathic pulmonary fibrosis. Lung 2005; 183: 291-300.

12 Renzoni EA, Walsh DA, Salmon M, et al. Interstitial vascularity in fibrosing alveolitis. Am J Respir Crit Care Med 2003; 167: 438-443.

13 Beaudry P, Force J, Naumov GN, et al. Differential effects of vascular endothelial growth factor receptor-2 inhibitor ZD6474 on circulating endothelial progenitors and mature circulating endothelial cells: implications for use as a surrogate marker of antiangiogenic activity. Clin Cancer Res 2005; 11: 3514-3522.

14 Jeon $\mathrm{K}$, Chung MP, Lee KS, et al. Prognostic factors and causes of death in Korean patients with idiopathic pulmonary fibrosis. Respir Med 2006; 100: 451-457. 
15 Martinez FJ, Safrin S, Weycker D, et al. The clinical course of patients with idiopathic pulmonary fibrosis. Ann Intern Med 2005; 142: 963-967.

16 Thabut G, Mal H, Castier Y, et al. Survival benefit of lung transplantation for patients with idiopathic pulmonary fibrosis. J Thorac Cardiovasc Surg 2003; 126: 469-475.

17 Shitrit D, Gershman Y, Peled N, et al. Risk factors for death while awaiting lung transplantation in Israeli patients: 1997-2006. Eur J Cardiothorac Surg 2008; 34: 444-448.

18 Nathan SD, Shlobin OA, Ahmad S, et al. Serial development of pulmonary hypertension in patients with idiopathic pulmonary fibrosis. Respiration 2008; 76: 288-294.

19 Nathan SD, Shlobin OA, Ahmad S, et al. Pulmonary hypertension and pulmonary function testing in idiopathic pulmonary fibrosis. Chest 2007; 131: 657-663.

20 Shorr AF, Wainright JL, Cors CS, et al. Pulmonary hypertension in patients with pulmonary fibrosis awaiting lung transplant. Eur Respir J 2007; 30: 715-721.

21 Nadrous HF, Pellikka PA, Krowka MJ, et al. The impact of pulmonary hypertension on survival in patients with idiopathic pulmonary fibrosis. Chest 2005; 128: Suppl. 6, 616S-617S.

22 Mejia M, Carrillo G, Rojas-Serrano J, et al. Idiopathic pulmonary fibrosis and emphysema: decreased survival associated with severe pulmonary arterial hypertension. Chest 2009; 136: 10-15.

23 Collard HR, Anstrom KJ, Schwarz MI, et al. Sildenafil improves walk distance in idiopathic pulmonary fibrosis. Chest 2007; 131: 897-899.
24 Zisman DA, Schwarz M, Anstrom KJ, et al. A controlled trial of sildenafil in advanced idiopathic pulmonary fibrosis. $N$ Engl J Med 2010; 363: 620-628.

25 Daniels CE, Yi ES, Ryu JH. Autopsy findings in 42 consecutive patients with idiopathic pulmonary fibrosis. Eur Respir J 2008; 32: 170-174.

26 Parra ER, Silverio da Costa LR, Ab'Saber A, et al. Nonhomogeneous density of CD34 and VCAM-1 alveolar capillaries in major types of idiopathic interstitial pneumonia. Lung 2005; 183: 363-373.

27 Colombat M, Mal H, Groussard O, et al. Pulmonary vascular lesions in end-stage idiopathic pulmonary fibrosis: histopathologic study on lung explant specimens and correlations with pulmonary hemodynamics. Hum Pathol 2007; 38: 60-65.

28 Pietra GG, Capron F, Stewart S, et al. Pathologic assessment of vasculopathies in pulmonary hypertension. J Am Coll Cardiol 2004; 43: Suppl. 12, 25S-32S.

29 Weitzenblum E, Chaouat A. Pulmonary hypertension due to chronic hypoxic lung disease. In: Peacock AJ, Rubin LJ, eds. Pulmonary Circulation: Diseases and Their Treatment. London, Arnold, 2004; pp. 374-386.

30 Galie N, Hoeper MM, Humbert M, et al. Guidelines for the diagnosis and treatment of pulmonary hypertension. Eur Respir J 2009; 34: 1219-1263.

31 Janda S, Shahidi N, Gin K, et al. Diagnostic accuracy of echocardiography for pulmonary hypertension: a systematic review and meta-analysis. Heart 2011; 97: 612-622.

32 Goodman Gilman AG, Rall TW, Nies AS, et al., eds The Pharmacological Basis of Therapeutics. 8th Edn. New York, Macmillan, 1990. 